\title{
Influence of Sample Size and Magnetizing Voltage on Barkhausen Noise during Bending and Uniaxial Tensile Tests
}

\author{
J. ŠrámeK*, M. Neslušan, F. Bahleda, K. Zgútová and P. Schenk \\ University of Žilina, Univerzitná 1, 01026 Žilina, Slovakia
}

\begin{abstract}
This paper reports about influence of sample thickness and magnetizing voltage on the Barkhausen noise emission during bending as well as uniaxial tensile tests. This study demonstrates that the Barkhausen noise in the tensile stress loading for the low sample thickness and low magnetizing voltage decreases. Such behaviour is considered to be a result of predominating stress anisotropy whereas the crystal anisotropy influence is only minor (or missing). For this reason, the conventional effective value of the Barkhausen noise signal cannot be employed for non-destructive monitoring of stress state in such a case and the magnitude of exerted stresses should be calibrated the width of the Barkhausen noise envelope. It was also found that the sample thickness as well as the manner of exerted loading also play significant roles since the Barkhausen noise tends to increase with increasing tensile stresses, especially for the higher magnetizing fields.
\end{abstract}

DOI: 10.12693/APhysPolA.137.640

PACS/topics: stress, Barkhausen noise, magnetizing

\section{Introduction}

When steel bridges are exposed to the mechanical load, cyclic fluctuation occurs as a result of the real operation as well as the superimposing corrosion attack. Monitoring of corrosion micro or/and macro cracking due to long term bridge operation has been a challenging task for many years. It was also found that magnetic Barkhausen noise (MBN) technique is sensitive to the exerted stresses in the real bridges [1], corrosion extent [2], as well as microcracking. For this reason, this technique could be employed for the aforementioned purpose. However, it is necessary to distinguish between the influence of exerted stress state and microstructure (especially its alteration due to corrosion and superimposing cracking). Influence of stress state on MBN can be investigated directly on the real bridges [1] or in the laboratory on the mechanically loaded samples or/and a bridge models. These models (or samples) are usually made of components of thin walls and calibration of MBN versus loaded stress can be carried out with bending or uniaxial tensile tests. Such investigation should be carried out before the degradation process to access (or unwrap) the contribution of the different aspects contributing to MBN. Therefore, this study reports about calibration of MBN versus stress and discusses the specific behaviour of MBN evolution for samples made of thin walls.

\section{Experiments}

The experimental study was carried out on the low carbon hot rolled steel S460 MC of $A=26.3 \%$,

*corresponding author; e-mail: juraj.sramek@fstav.uniza.sk
$R_{m}=610 \mathrm{MPa}, R_{p 0.2}=540 \mathrm{MPa}(0.082 \% \mathrm{C}, 1.04 \% \mathrm{Mn}$, $0.02 \mathrm{Si})$. This steel is employed for steel bridges and is subjected to the long term stress and superimposing corrosion attack. The samples of size $200 \times 22 \times 5$ and $200 \times 22 \times 3 \mathrm{~mm}^{3}$ were applied. MBN was measured by the use of RollScan 350 (magnetizing voltage 2, 3.5, 5, and $8 \mathrm{~V}$, magnetizing frequency $125 \mathrm{~Hz}$, frequency range of MBN $10-1000 \mathrm{kHz}$ ). MBN refers to the root-meansquare (rms, effective) value of the signal. Number of detected MBN pulses, peak position (PP), and full width of half maximum (FWHM) of MBN envelopes were also extracted.

Influence of stresses was investigated in the elastic regime of loading up to $300 \mathrm{MPa}$ which is far below the yield stress. Two different ways of sample loading were carried out. Tensile stresses (TS) as well as compressive stresses (CS) $\pm 300 \mathrm{MPa}$ were exerted during the bending test whereas only TS were generated during the uniaxial tensile test.

The bending test was operated in the self-made system. Stress state was measured via strain gauges technique on the opposite side of the sample as Fig. 1 illustrates. Uniaxial tensile test was carried out by the use of Instron 5985 device and stress state was checked directly by setting up the loading force.

\section{Results of experiments}

RollScan 350 Barkhausen noise analyser provides automated magnetization voltage and frequency sweeps. These sweeps can be used for monitoring surfaces [3]. As Thomas and Fix [4] reported, performing these sweeps on samples of varying properties can provide much insight when optimizing a system for maximum sensitivity. When measuring MBN for measuring stress state magnetizing the samples into saturation can decrease the sensitivity or the measured difference between the varying 


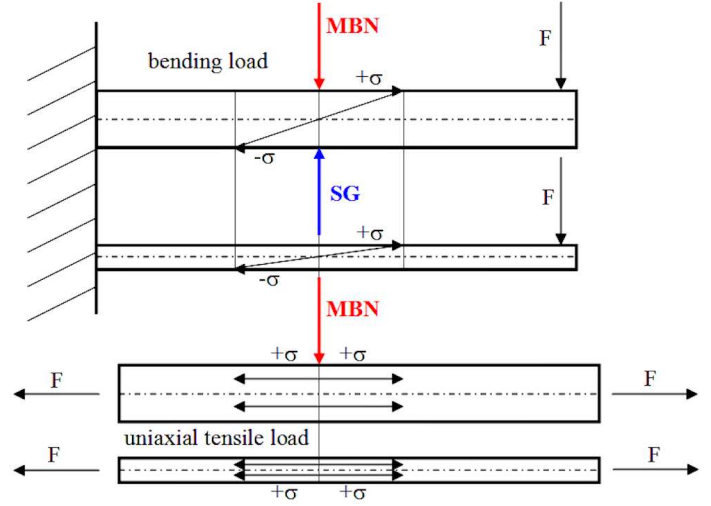

Fig. 1. Brief sketch of the samples loading.

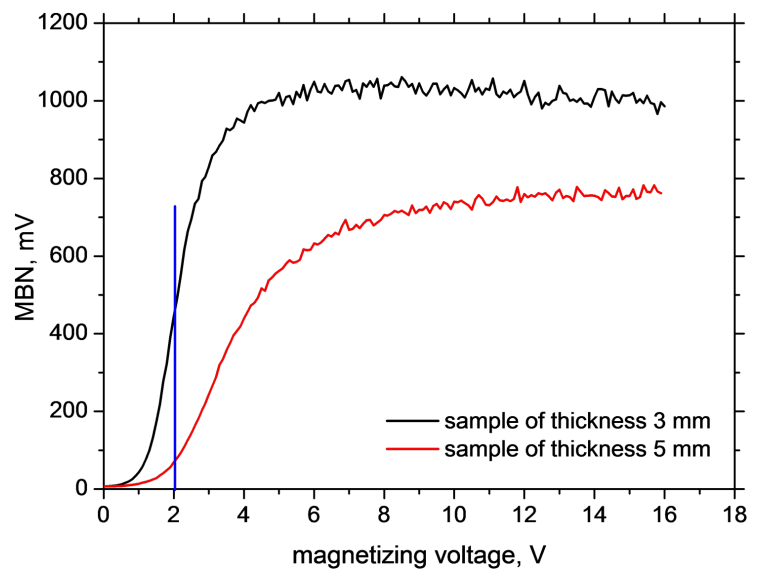

Fig. 2. Voltage sweeps.

stress states [4]. This occurs because mechanical stresses alter the magnetostrictive energy of a sample, thus affecting the coercivity and remanence of the sample. This is known as the Villari effect [5]. For this reason it is critical that samples are not magnetically saturated and it is recommended [6] to set up the magnetizing voltage in the position of the steepest part in the magnetizing voltage sweep.

Figures 2 and 3 depict that the magnetizing sweeps are also functions of the sample thickness. On the one hand, the magnetizing frequency of $125 \mathrm{~Hz}$ can be applied for both samples. On the other hand, the optimal magnetizing voltages for the samples of the different thickness differ. $2 \mathrm{~V}$ should be the optimal voltage for the sample of $3 \mathrm{~mm}$ thickness and $3.5 \mathrm{~V}$ for sample of $5 \mathrm{~mm}$ thickness. Therefore, keeping the magnetizing frequency constant at $125 \mathrm{~Hz}$, the different magnetizing voltages up to saturation of the samples were investigated $(2,3.5,5$, and $8 \mathrm{~V}$ ). The differences in the appearance of magnetizing sweeps are due to the higher magnetic flux density for the thinner sample. For this reason, the voltage sweep for the thinner sample increases and saturates earlier with respect to the magnetizing voltage. Moreover, MBN values for the thinner sample can be found to be higher since

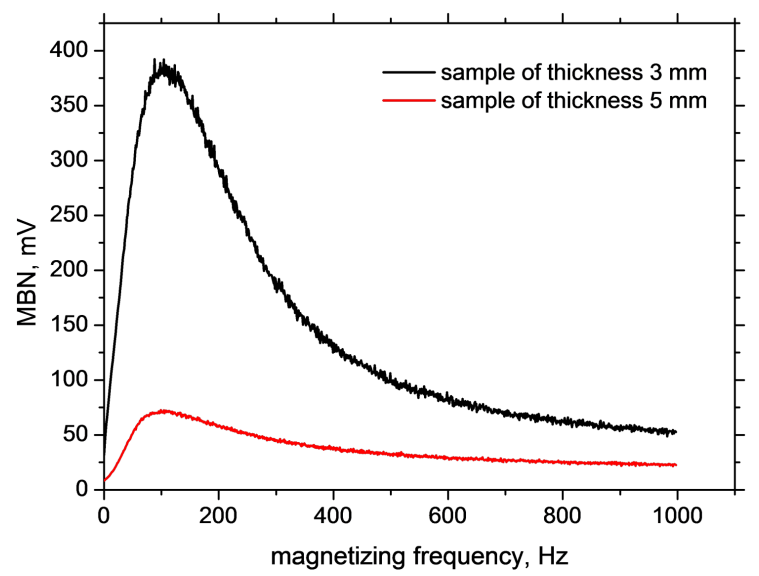

Fig. 3. Frequency sweeps.

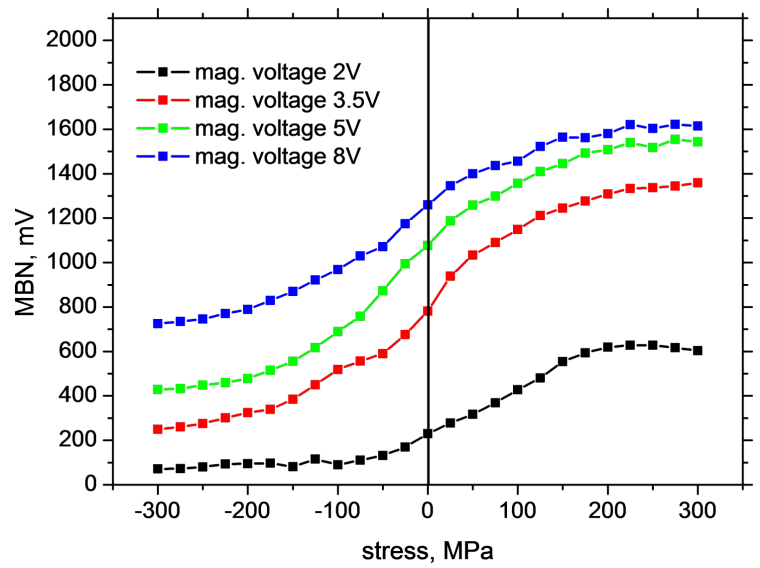

Fig. 4. Stress state versus MBN, bending test - sample thickness $5 \mathrm{~mm}$.

such effect contributes to the stronger magnetizing field in the thinner sample as compared to the thicker one. By changing the amplitude of magnetizing voltage, the $\mathrm{d} H / \mathrm{d} t$ is changing and this factor takes important role in MBN evolution as well. This effect is similar as for changing the frequency at constant amplitude.

Figures 4 and 5 demonstrate that this effect also alters evolution of MBN versus applied stress. Figure 4 shows the typical evolution of MBN as a function of stress state in which MBN decreases with CS and increases with TS due to the well-known effect of the domain walls alignment. However, the relationship between the TS and MBN for the thinner sample differs. MBN decreases with TS for magnetizing voltage of $2 \mathrm{~V}$ and exhibits early saturation for the higher magnetizing voltages. Such behaviour has been already reported by Amiri et al. [7]. As the authors reported, there is a competition between two anisotropies to determine the magnetization easy axis under applied stress. At low applied stress, crystal anisotropy plays the main role on the magnetization process and MBN increases, while at higher stresses, stress anisotropy plays main role and MBN decreases. 


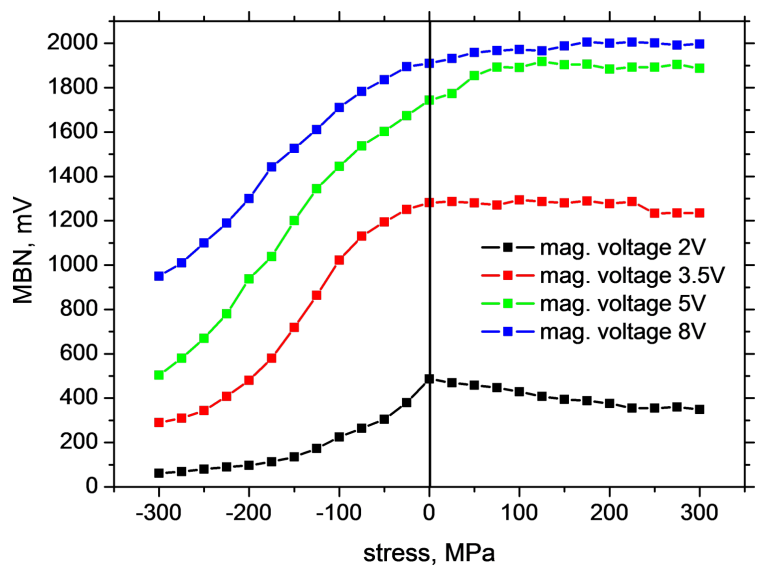

Fig. 5. Stress state versus MBN, bending test - sample thickness $3 \mathrm{~mm}$.

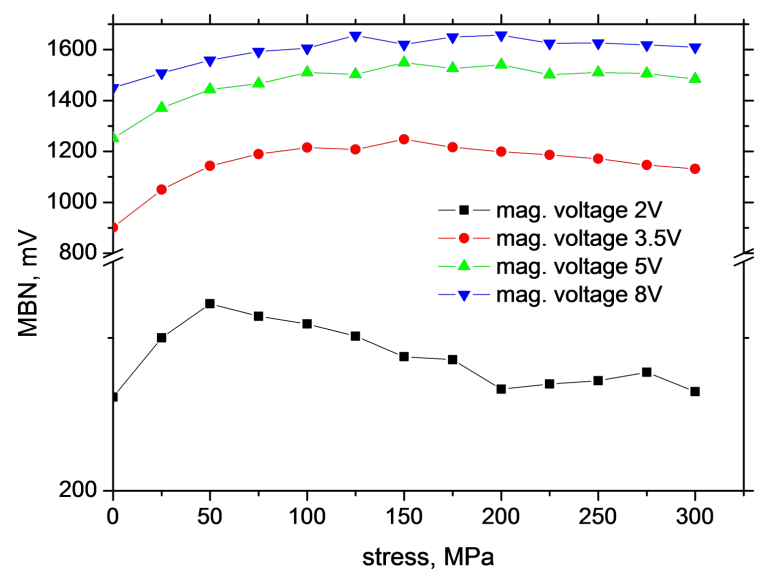

Fig. 6. Stress state versus MBN, uniaxial tensile test - sample thickness $3 \mathrm{~mm}$.

This means that in the case of the lower tensile stresses the easy axis is controlled by crystal anisotropy. Thus domains and the corresponding domain walls turn into direction of the magnetic easy axis. On the other hand, at the higher TS the domain and domain walls are forced to turn into direction of the new easy axis which is controlled by stress [7]. Such behaviour can be seen in Fig. 4 for magnetizing voltage of $2 \mathrm{~V}$ whereas ascending part of MBN evolution for thinner sample and the same voltage is fully missing (see Fig. 5).

Comparison of Figs. 5 and 6 demonstrates that the manner of exerted loading also plays significant role since MBN tends to increase with increasing TS especially for the higher magnetizing voltages and evolution of MBN with TS proves the findings reported by Amiri et al. [7]. The main difference between the bending and the uniaxial tensile test is associated with the distribution of TS within the MBN sensing depth. Magnitude of TS decreases with increasing depth from the free surface, while uniform TS distribution can be found for the uniaxial tensile test.

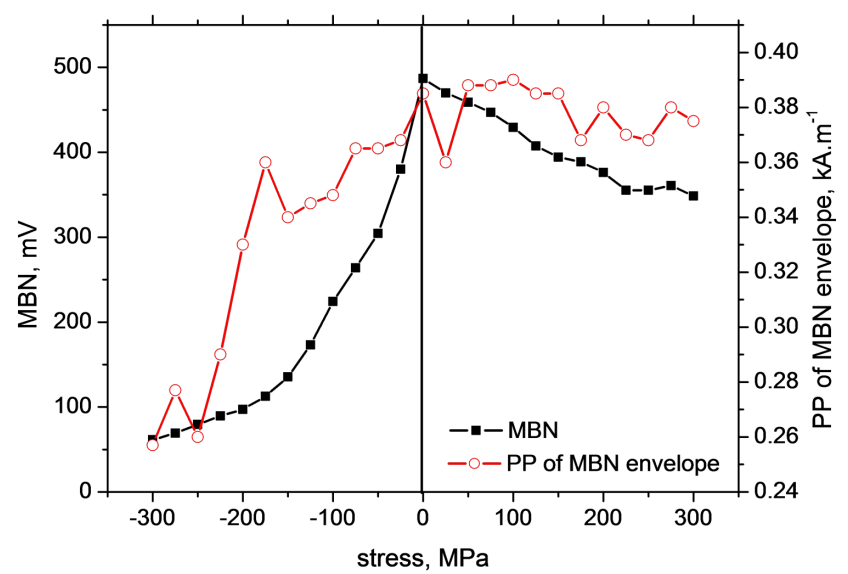

Fig. 7. MBN and PP of MBN envelope versus applied stress.

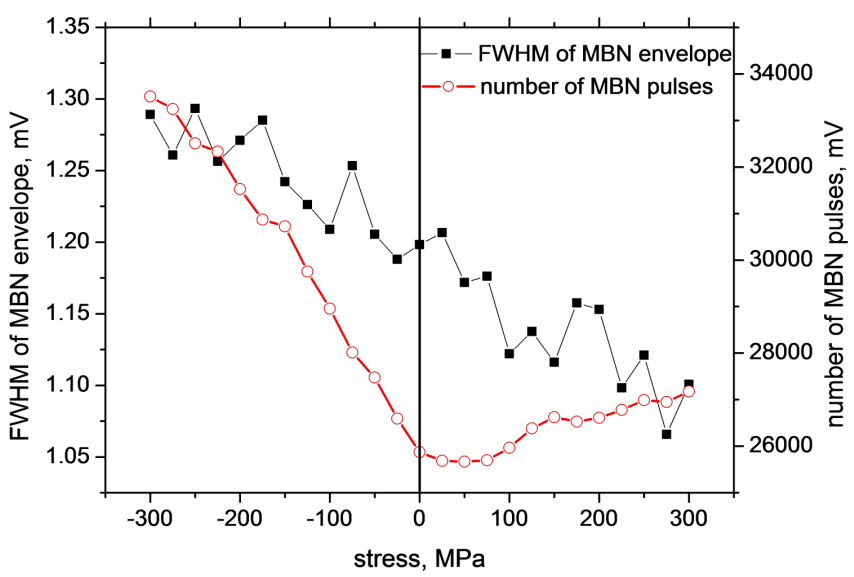

Fig. 8. Number of MBN pulses and FWHM of MBN envelope versus applied stress.

Figures 7 and 8 illustrate that it is not possible to distinguish between TS and CS by the use of MBN, PP, or MBN pulses. PP progressively increases with decreasing magnitude of CS and saturates in the TS region whereas evolution of MBN pulses is inversely proportional to the MBN evolution. Only FWHM could be found as an alternative since Fig. 8 shows the linear decrease through the applied stresses.

\section{Conclusions}

This study demonstrates that the relationship between MBN and TS for the components made of thin samples is very flat and the FWHM of MBN envelope can be used only as the alternative parameter for assessment of stress state. The preliminary phase in which the optimized magnetizing conditions are proposed should take into consideration not only evolution of MBN against the magnetizing voltage but also associated sensitivity of MBN against exerted stresses. 


\section{Acknowledgments}

This work was supported by the VEGA projects no. $1 / 0170 / 17$ and no. $1 / 0121 / 17$.

\section{References}

[1] K. Zgútová, M. Neslušan, K. Kolařík, J. Šrámek, in: Proc. 7th Int. Conf. on Mechanics and Materials in Design, M2D2017, Albufeira (Portugal), 2017.

[2] M. Neslušan, F. Bahleda, P. Minárik, K. Zgútová, M. Jambor, J. Magn. Magn. Mater. 484, 179 (2019).
[3] S. Santa-aho, M. Vippola, A. Sorsa, K. Leiviskä, M. Lindgren, T. Lepistö, NDTE Int. 52, 95 (2012).

[4] J. Thomas, R. Fix, in: Proc. ICBM-7 Conf., Aachen (Germany), 2009.

[5] J.D. Griffiths, Introduction to Electrodynamics, Prentice Hall, NJ 1999.

[6] MicroScan 600 - Operating instructions manual V.5.4b (2015-0-06), Stresstech Group, Finland.

[7] M.S. Amiri, M. Thielen, M. Rabung, M. Marx, K. Szielasko, Ch. Boller, J. Magn. Magn. Mater. 372, 16 (2014). 